\title{
Frauen in der beruflichen Selbständigkeit
}

\section{Eine erfolgversprechende Alternative zur abhängigen Beschäftigung?*}

\author{
Monika Jungbauer-Gans und Peter Preisendörfer \\ Institut für Soziologie, Ludwig-Maximilians-Universität München, Konradstr. 6, D-8000 München 40
}

\begin{abstract}
Zus a mm enf as sung: Der Beitrag beschäftigt sich mit der Frage, ob - im Sinne einer individuellen Handlungsstrategie - die berufliche Selbständigkeit für Frauen eine erfolgversprechende Alternative zur abhängigen Beschäftigung ist, d.h. eine Möglichkeit, um objektiv bestehenden oder subjektiv wahrgenommenen Diskriminierungen auf dem allgemeinen Arbeitsmarkt auszuweichen. Als empirische Datenbasis dient die „Münchner Gründerstudie“, eine Befragung von rund 1850 Unternehmensgründerinnen und -gründern. Die wichtigsten Ergebnisse sind: Frauen treten zum einen mit einer ungünstigeren Humankapitalausstattung als Männer in die berufliche Selbständigkeit ein und gründen zum anderen Betriebe, die eher als randständige Selbständigkeitsexistenzen gesehen werden müssen. Die Folge ist, daß „Frauenbetriebe“ (im Vergleich zu „Männerbetrieben“) im Aggregat geringere Überlebenschancen haben und insgesamt auch weniger „expansiv" sind. Würden jedoch die Frauen mit denselben Humankapitalressourcen wie die Männer in die Selbständigkeit eintreten und/oder die gleiche Art von Betrieben gründen, gäbe es keine geschlechtsspezifischen Unterschiede der Bestands- und Überlebenschancen der Betriebe. Dieses - im Unterschied zu abhängigen Beschäftigungsverhältnissen - Fehlen eines eigenständigen Geschlechtseffekts bei Kontrolle der wichtigsten ,anderen“ Faktoren wird dergestalt interpretiert, daß mit der Entscheidung für die berufliche Selbständigkeit für die Frauen einige Hürden entfallen, die ihre Chancen auf dem allgemeinen Arbeitsmarkt beschränken.
\end{abstract}

\section{Einleitung}

$\mathrm{Daß}$ es auf dem Arbeitsmarkt (noch immer) massive geschlechtsspezifische Ungleichheiten gibt, läßt sich - abgesehen von der Primärerfahrung - durch eine lange Serie empirischer Forschungsergebnisse belegen. Sehr viel weniger gut erforscht sind jedoch die individuellen und kollektiven Handlungsmöglichkeiten für Frauen (und Männer), die mit Blick auf das Ziel einer Angleichung der geschlechtsspezifischen Differenzen erfolgversprechend erscheinen. Zum Spektrum der kollektiven Handlungsstrategien gehören z. B. gesetzliche Regelungen, unternehmenszentrierte Programme oder auch eine verbesserte interne Organisation der Fraueninteressen. Individuelle Handlungsmöglichkeiten von Frauen beziehen sich auf die Berufswahlentscheidung (Stichwort: Frauen in Männerberufen), Arbeitsplatzwechsel, gegebenenfalls das „Timing“ von Erwerbsunterbrechungen oder auch die juristische Durchsetzung von Ansprüchen.

Der vorliegende Artikel untersucht eine der möglicherweise erfolgversprechenden individuellen Handlungsstrategien von Frauen, nämlich die Op-

\footnotetext{
* Der Artikel entstand im Rahmen der Arbeit der Autorin und des Autors in dem von der Deutschen Forschungsgemeinschaft geförderten Projekt „Berufserfahrung und Erfolgschancen von Unternehmensgründern“ (Zi 207/7-2, Projektleiter: Rolf Ziegler).
}

tion der beruflichen Selbständigkeit. Bedeutsam erscheint diese Strategie vor dem Hintergrund folgender Informationen und Überlegungen: Wenngleich Frauen im Bereich der beruflichen Selbständigkeit traditionell unterrepräsentiert sind, läßt sich seit Beginn der 70er Jahre eine wachsende Tendenz beobachten, daß Frauen in die Selbständigenrolle eintreten bzw. überwechseln (vgl. dazu z. B. Assig et al. 1985; Ambos 1989; Bühler 1991). Dies mit dem Ergebnis, daß sich, wenngleich zögerlich, ihre Unterrepräsentanz bei den Selbständigen abschwächt. Mit dem Wegfallen des Arbeitgebers und des betrieblichen Kontexts entfallen gleichzeitig eine Reihe von Mechanismen, über die Frauen in der Regel auf schlechter bezahlte Positionen, auf Positionen mit geringeren Aufstiegschancen, Entscheidungsbefugnissen usw. verwiesen werden. Schließlich eröffnet die Selbständigenrolle, zumindest im Prinzip, von der Zeitgestaltung her eher die Möglichkeit, eigenständig die Anforderungen von Erwerbsarbeit und Familie aufeinander abzustimmen. Mit letzterem Argument erscheint die berufliche Selbständigkeit für Frauen nicht nur als eine Alternative zur abhängigen Beschäftigung, d.h. um objektiv bestehenden oder subjektiv wahrgenommenen Diskriminierungen auf dem allgemeinen Arbeitsmarkt auszuweichen, sondern gleichzeitig eventuell auch als eine Alternative zur vielbeschworenen "Nur-Hausfrau“.

Ob nun aber die berufliche Selbständigkeit für Frauen tatsächlich eine erfolgversprechende Alter- 
native ist, muß im einzelnen noch genauer untersucht werden. Bislang liegen dazu nur recht wenige empirische Befunde vor (vgl. u. a. Schlemper-Kubista/Wollrab 1980; Wloch/Ambos 1986; Assig 1987; Wloch/Siegel 1988; Ambos 1989; Kirsch/Lühder 1991). Die wenigen vorliegenden empirischen Evidenzen sind zudem in der Regel mit mehr oder weniger gravierenden Mängeln behaftet: Die Ergebnisse basieren oft auf sehr kleinen Stichproben; überwiegend ist die Untersuchungsgruppe mit einem ,Survivor-Bias“ belastet, d. h. es werden eher erfolgreiche, schon längere Zeit bestehende $\mathrm{Be}$ triebe betrachtet; und weiterhin fehlt zumeist eine Kontrollgruppe männlicher Selbständiger. ${ }^{1}$

Die Frage der Selbständigkeit als erfolgversprechende Alternative für Frauen soll im vorliegenden Artikel dergestalt angegangen werden, daß untersucht wird, ob und inwieweit sich die weithin bekannten geschlechtsspezifischen Unterschiede auf dem allgemeinen Arbeitsmarkt auch im Bereich der beruflichen Selbständigkeit wiederfinden. Mit dieser Wendung der Fragestellung soll einerseits - im Unterschied zur bisherigen Forschung - explizit die Notwendigkeit einer männlichen Kontrollgruppe betont werden, andererseits kann relativ bruchlos an die Ergebnisse der Forschungstradition zur geschlechtsspezifischen Ungleichheit im Bereich der abhängigen Beschäftigung angeknüpft werden. Sollte sich herausstellen, daß im Bereich der beruflichen Selbständigkeit ähnliche geschlechtsspezifische Disparitäten bestehen wie im Bereich der abhängigen Beschäftigung, kann die Selbständigkeitsoption wohl kaum als eine erfolgversprechende Alternative für Frauen eingestuft werden.

Als Untersuchungsgruppe für unsere empirischen Analysen dient eine Kohorte von rund 1850, in den Jahren 1985/86 im Raum München und Oberbayern neugegründeten Betrieben, deren Gründer/innen Anfang 1990 interviewt wurden („Münchner Gründerstudie“). Konkret soll die Ausgangsfragestellung in fünf Untersuchungsschritte aufgegliedert werden, wobei stets die Unterschiede zwischen „Frauen-“ und „Männerbetrieben“ im Mit-

\footnotetext{
${ }^{1}$ Neben den wenigen empirischen Studien, die sich direkt mit Frauen in der beruflichen Selbständigkeit befassen, gibt es eine Reihe weiterer Studien zư „Karrieremustern erfolgreicher Frauen", wobei diese Frauen zum Teil ebenfalls beruflich selbständig sind (vgl, z. B. Bock-Rosenthal et al. 1978; Metzler 1986). Auch diese Studien stützen sich zumeist auf kleine Stichproben und haben damit eher explorativen Charakter.
}

telpunkt des Interesses stehen: (1) Gibt es Unterschiede in der individuellen Humankapitalausstattung von Betriebsgründerinnen und -gründern, also Unterschiede in den personellen Voraussetzungen und Ressourcen, die von weiblichen und männlichen Gründungspersonen in den Betrieb eingebracht werden? (2) Inwieweit unterscheiden sich Frauen und Männer hinsichtlich der Art der von ihnen gegründeten Betriebe, d.h. gibt es geschlechtsspezifische Differenzen z. B. bei der GröBe oder der Wirtschaftszweigzugehörigkeit der Betriebe? (3) Haben Frauen- und Männerbetriebe unterschiedliche Bestands- und Überlebenschancen? (4) Unterscheiden sich Frauen- und Männerbetriebe, sofern sie Bestand haben, in ihrer Entwicklung im Zeitablauf, und zwar speziell bei der Entwicklung der Zahl der Beschäftigten und bei der Umsatzentwicklung? (5) Das Bestandskriterium und die Entwicklung der Beschäftigten und des Umsatzes können als „harte“ Erfolgsindikatoren für betriebliche Neugründungen gelten. Im fünften Untersuchungsschritt soll schließlich noch ein Blick auf einige ergänzende, zum Teil auch subjektive Erfolgsmaße der Gründungen geworfen werden.

Überblickt man diesen Satz von Fragestellungen, könnte - in Anknüpfung an das klassische Vorurteil - die Leitfrage unserer Arbeit stark vereinfachend auch in die Form gebracht werden: „Sind Frauen die schlechteren Unternehmer?" Da wir uns in allen Punkten auf die im Grundtenor geläufigen Befunde der Forschung über Ausmaß, Art und Ursachen der geschlechtsspezifischen Ungleichheit auf dem allgemeinen Arbeitsmarkt stützen können (für neuere Beiträge z. B. Autorinnengemeinschaft 1989, 1990; Marini 1989; Mayer et al. 1991), können die Ausgangshypothesen unserer Analysen jeweils im Zusammenhang mit der Darstellung der empirischen Ergebnisse erläutert werden, so daß auf einen eigenständigen, theoretischen Abschnitt verzichtet wird. Bevor aber die fünf angezielten Fragestellungen untersucht werden können, muß zunächst unsere empirische $\mathrm{Da}$ tenbasis erläutert werden.

\section{Empirische Datenbasis}

Empirische Grundlage für unsere Analysen der Unterschiede von neugegründeten Frauen- und Männerbetrieben sind die Daten der ,Münchner Gründerstudie“. Bei dieser Studie handelt es sich um eine Anfang 1990 durchgeführte mündliche Befragung von Personen, die in den Jahren 1985/86 
im Kammerbezirk der Industrie- und Handelskammer für München und Oberbayern ein Gewerbe angemeldet hatten. Aus der Grundgesamtheit aller Gewerbeanmeldungen der Jahre 1985/86 im IHKBezirk München und Oberbayern (knapp 30000 Meldungen) wurde eine geschichtete Zufallsstichprobe von rund 6000 Meldungen gezogen. ${ }^{2}$ Betriebe, die zum Befragungszeitpunkt bereits wieder abgemeldet waren, wurden in der Stichprobe übergewichtet, da von vorneherein zu erwarten war, daß bei diesen Gründerinnen und Gründern die Bereitschaft zur Mitwirkung an der Befragung niedriger sein wird.

In der ersten Phase der Studie mußten zunächst die Adressen der 6000 Stichprobenfälle aktualisiert werden. Da sich das von der IHK bereitgestellte Adressenmaterial auf die Angaben bezog, die die Gründer/innen bei ihrer Gewerbeanmeldung in den Jahren 1985/86 gemacht hatten, mußte davon ausgegangen werden, daß zahlreiche Anschriften nicht mehr aktuell waren. Im Endeffekt konnten von den 6000 Startadressen rund $90 \%$ aktualisiert werden. Im zweiten Schritt mußten die Betroffenen motiviert werden, an der Befragung teilzunehmen. Dies geschah durch zwei Anschreiben und gegebenenfalls durch zusätzliche Telefonanrufe. Im Zeitraum von Januar bis Mai 1990 konnten insgesamt 481 Gründerinnen (26\%) und 1368 Gründer (74\%) befragt werden. Bezieht man die 1849 Befragten auf die 6000 Startadressen der Stichprobe, erscheint die Erfolgsquote von 31\% gemessen an dem, was man für Betriebsbefragungen in der Regel erwartet - auf den ersten Blick durchschnittlich. Allerdings ist zu berücksichtigen, $\mathrm{daB}$ das, was sich hinter Gewerbeanmeldungen im einzelnen verbirgt, sehr vielfältig und heterogen ist. Für fast $20 \%$ unserer Startadressen erwies sich, $\mathrm{daB}$ die ausgewählten Personen bzw. Betriebe nicht zur Zielgruppe unserer Studie gehörten (z. B. weil trotz Gewerbeanmeldung nie eine Betriebsaktivität zustande kam oder weil es sich um einen schon lange bestehenden Betrieb handelte, bei dem es lediglich zu einer formalen, rechtlichen Veränderung kam).

Das inhaltliche Frageprogramm der weitgehend standardisierten Interviews war sehr umfangreich,

${ }^{2}$ Die Beschränkung der Grundgesamtheit auf Gewerbemeldungen impliziert, $\mathrm{da} B$ Handwerksbetriebe und freiberufliche Tätigkeiten (Ärzte, Rechtsanwälte usw.) ausgeschlossen sind. Für die Bereitstellung der Gewerbemeldedaten möchten wir an dieser Stelle der IHK für München und Oberbayern und dabei vor allem Herrn Dr. Zipperlen unseren Dank aussprechen. so $\mathrm{da} ß$ eine Interviewzeit von durchschnittlich rund 45 Minuten notwendig war. Im ersten Teil des Fragebogens wurden zahlreiche Basismerkmale des Betriebes und der betrieblichen Entwicklung (gegebenenfalls auch Gründe für die Abmeldung des Betriebes) erfragt. Im zweiten Teil wurde detailliert auf die Person der Gründerin bzw. des Gründers eingegangen (ausführlichere Informationen zur Münchner Gründerstudie finden sich in Preisendörfer/Ziegler 1990).

\section{Humankapitalausstattung von Unternehmensgründerinnen und -grïndern}

Eine der Ursachen der in zahlreichen empirischen Studien festgestellten geschlechtsspezifischen Disparitäten auf dem Arbeitsmarkt ist die - in der Terminologie der ökonomischen Humankapitaltheorie (Mincer 1974; Becker 1975) - ungleiche Humankapitalausstattung von Frauen und Männern. Obwohl der Begriff des Humankapitals im einzelnen sehr weit gefaßt werden kann (Gesundheit, verschiedene „abilities“ usw.), umfaßt das Konzept im Rahmen der gängigen, meist auf abhängige Beschäftigungsverhältnisse zugeschnittenen Operationalisierungen in der Regel zumindest die Bildungsinvestitionen einer Person sowie die Dauer der Berufserfahrung. Bezüglich der Verteilung der Bildungsabschlüsse ist bekannt, daß die Frauen im Zuge der Bildungsexpansion deutlich aufholen konnten (dazu z. B. Blossfeld 1985, 1989; Handl 1986). Im Aggregat der aktuell Erwerbstätigen besteht gleichwohl noch immer ein Bildungsvorsprung der Männer. Weiterhin absolvieren junge Frauen seltener Berufsausbildungen im dualen System, häufiger jedoch sog. Schul-Berufsausbildungen, die bei höheren Voraussetzungen und höherem Aufwand zu geringeren Karrieremöglichkeiten führen (Krüger 1989). Hinsichtlich der Berufserfahrung können wir zunächst davon ausgehen, daß sich die Berufsbiographien von Frauen vor allem in den Übergangshäufigkeiten von Erwerbs- und Nichterwerbsarbeit - im Durchschnitt wechselvoller gestalten als die von Männern (dazu z.B. Hellmich 1986; Tölke 1989; Mayer 1991). Dies mit dem Ergebnis, daß Frauen aufgrund häufigerer Unterbrechungen ihrer Erwerbstätigkeit weniger allgemeine Berufserfahrung akkumulieren. Potentielle Erwerbsunterbrechungen antizipierend, gewähren Arbeitgeber schließlich Frauen seltener die Gelegenheit zum Erwerb sog. betriebsspezifischen Humankapitals (z. B. in Form von innerbetrieblichen Aus- und Weiterbildungen 
oder von Gelegenheiten zu „learning-on-the-job“) (dazu z. B. Lappe 1981; Gärtner/Krebsbach-Gnath 1987).

Überträgt man diese aus der allgemeinen Arbeitsmarktforschung geläufigen Befunde auf den Bereich der beruflichen Selbständigkeit, ergibt sich die Frage, ob Frauen auch als Unternehmerinnen aufgrund ihrer Humankapitalausstattung mit „schlechteren Karten“ in die berufliche Selbständigkeit eintreten. Bei der Untersuchung dieser Frage sollte man allerdings nicht nur die herkömmlichen Indikatoren (Bildung und Berufserfahrung) der Humankapitalausstattung berücksichtigen, sondern auch Aspekte eines spezifischen, unternehmerischen Humankapitals. Damit sind individuelle Ressourcen gemeint, die sich speziell für die gewählte Rolle des Selbständigen als nützlich erweisen dürften (ausführlicher zum Konzept des unternehmerischen Humankapitals z. B. Preisendörfer/Voss 1990; Brüderl et al. 1991). Wichtige Aspekte des spezifischen, unternehmerischen Humankapitals dürften vor allem vorherige berufliche Erfahrungen in der Branche der jeweiligen Gründung sein, eigene frühere Selbständigkeitserfahrungen oder auch Erfahrungen mit der beruflichen Selbständigkeit im elterlichen Herkunftsmilieu. Die Bedeutung vorheriger Branchenerfahrung der Gründungsperson für die Erfolgschancen eines Betriebes wird vor allem in der umfangreichen „Ratgeber"-Literatur zur beruflichen Selbständigkeit (,Wie mache ich mich selbständig?“) betont (dazu statt vieler Dittrich 1985; Hofmann 1986; speziell für Frauen: Assig 1987; Markmann 1988). Frühere, eventuell auch fehlgeschlagene Versuche zur beruflichen Selbständigkeit erscheinen wichtig, wenn man den Weg in die Selbständigkeit als eine Art von „Versuchs- und Irrtums-Verfahren“ begreift. Elterliche Selbständigkeit vermittelt Kenntnisse und Erfahrungen mit der Selbständigenrolle quasi als Nebenprodukt täglicher Interaktionen und kann mithin aus der Perspektive der Humankapitaltheorie die Entscheidung für die Selbständigkeit als rationale Handlungsstrategie nahelegen (dazu z. B. Laband/Lentz 1985).

Die wesentlichen Ergebnisse unserer Analysen zum Untersuchungsschritt der Humankapitalressourcen von Unternehmensgründerinnen und -gründern sind in Tabelle 1 festgehalten. Die empirischen Befunde hinsichtlich der allgemeinen $\mathrm{Hu}$ mankapitalausstattung entsprechen fast nahtlos und überraschend deutlich den Ausgangserwartungen, d.h. die geschlechtsspezifischen Unterschiede, die man aus dem Bereich abhängiger Beschäftigungsverhältnisse kennt, finden sich auch bei den
Betriebsgründerinnen und -gründern: Während nur $26 \%$ der untersuchten Gründerinnen Fachhochschulreife bzw. Abitur hatten, waren es bei den Gründern $43 \%$. Bei der in Jahren gemessenen Bildung (Jahre der schulischen und der beruflichen Ausbildung) beobachten wir eine Differenz von 1.3 Jahren. Obwohl die Frauen zum Zeitpunkt ihrer Betriebsgründung im Durchschnitt fast genauso alt waren wie die Männer (Durchschnittsalter der Frauen 35.9 gegenüber 36.5 Jahren bei den Männern), verfügten sie im Schnitt über 2.2 Jahre weniger Berufserfahrung. Dabei ist hinzuzufügen, $\mathrm{da} \beta$ die Dauer der Berufserfahrung in unserer Studie sehr genau erfaßt wurde, da detailliert die komplette bisherige Berufsbiographie aller Befragten - inklusive Erwerbsunterbrechungen - erhoben wurde. Frauen hatten ihre Erwerbstätigkeit im Durchschnitt 4.8 Jahre unterbrochen, Männer nur 1.2 Jahre. $^{3} 40 \%$ der Unternehmensgründerinnen (gegenüber $15 \%$ bei den Gründern) waren unmittelbar vor der Gründung ihres Betriebes nicht erwerbstätig, d. h. entweder als Hausfrau tätig oder arbeitslos. Arbeitslosigkeit als Gründungsmotiv spielte bei den untersuchten Gründerinnen und Gründern im Raum München und Oberbayern insgesamt eine eher untergeordnete Rolle. Nur $3.5 \%$ aller Befragten waren unmittelbar vor ihrer Betriebsgründung arbeitslos. $4.6 \%$ der Gründerinnen und $3.1 \%$ der Gründer gaben an, daß sie unmittelbar vor der Betriebsgründung arbeitslos waren. Eine oder mehrere Arbeitslosigkeitsepisoden im Verlauf der letzten zehn Jahre vor der Gründung hatten 15\% der Männer und 19\% der Frauen. Während unter den vor der Gründung erwerbstätigen Gründern 35\% unmittelbar vor der Gründung bereits selbständig bzw. freiberuflich tätig waren, waren es bei den erwerbstätigen Gründerinnen nur $23 \%$.

Ein sehr ähnliches Bild ergibt sich auch bei den von uns betrachteten Indikatoren des unternehmerischen Humankapitals: Die Frauen verfügten deutlich seltener über vorherige Branchen-, Selbständigkeits- und Führungserfahrung. ${ }^{4}$ Die zeitliche Dauer der beruflichen Erfahrung in der Bran-

${ }^{3}$ Die 1.2 Jahre Erwerbsunterbrechung bei den Männern kommen vor allem durch den Wehr- bzw. Zivildienst zustande. Zählt man diesen nicht als Erwerbsunterbrechung, vermindert sich die Unterbrechungszeit bei den Männern auf 0.6 Jahre.

4 Führungserfahrung bezieht sich darauf, ob die befragte Person in früheren beruflichen Positionen Untergebene hatte bzw. Mitarbeiter/innen zu leiten hatte. 
Tabelle 1 Humankapitalausstattung von Unterneh. mensgründerinnen und -gründern

$\begin{array}{ll}\text { Frauen } & \text { Männer } \\ (n=481) & (n=1368)\end{array}$

Höchster SchulabschluB**

\begin{tabular}{|c|c|}
\hline $\begin{array}{l}\text { Volks-/Hauptschule } \\
\text { Mittlere Reife } \\
\text { Fachhochschulreife } \\
\text { Abitur }\end{array}$ & $\begin{array}{r}37 \% \\
37 \% \\
4 \% \\
22 \%\end{array}$ \\
\hline Bildung in Jahren ${ }^{\star *}$ & 12.2 \\
\hline Berufserfahrung in Jahren ${ }^{\star \star}$ & 12.6 \\
\hline \multicolumn{2}{|c|}{ Berufliche/soziale Stellung ${ }^{* *}$} \\
\hline $\begin{array}{l}\text { Nicht erwerbstätig } \\
\text { erwerbstätig, darunter: } \\
\text { - Arbeiter } \\
\text { - Angestellte, Beamte } \\
\text { - Selbständige, Freiberufler }\end{array}$ & $\begin{array}{r}40 \% \\
60 \% \\
3 \% \\
74 \% \\
23 \%\end{array}$ \\
\hline Branchenerfahrung ${ }^{\star *}$ & $50 \%$ \\
\hline Selbständigkeitserfahrung ** & $19 \%$ \\
\hline Führungserfahrung & $46 \%$ \\
\hline Selbständiger Vater & $38 \%$ \\
\hline
\end{tabular}

** Unterschied zwischen den Subgruppen (Frauen-Männer) ist signifikant auf dem $1 \%$-Niveau; aufgrund fehlender Werte bei den Aufgliederungsmerkmalen vermindern sich in den beiden Subgruppen zum Teil die Fallzahlen geringfügig.

che der Gründung (sowohl als abhängig Beschäftigte als auch als Selbständige) belief sich bei den Männern auf 7.9 Jahre, bei den Frauen auf 5.2 Jahre. Lediglich bei der elterlichen Selbständigkeit, gemessen am beruflichen Status des Vaters, ergibt sich kein signifikanter geschlechtsspezifischer Unterschied, d.h. die Gründerinnen kommen ebenso häufig aus einem Selbständigenhaushalt wie die Gründer.

Die aufgeführten Befunde belegen nun sicherlich noch nicht, daß berufliche Selbständigkeit für Frauen keine erfolgversprechende Alternative ist oder daß Frauen die schlechteren Unternehmer sind. Gleichwohl muß festgestellt werden, daß die geschlechtsspezifischen Unterschiede weitgehend analog zu den bekannten Unterschieden im Bereich der abhängigen Beschäftigung sind. Orientiert man sich zudem an den Erfahrungen vorliegender empirischer Studien der Gründungsforschung, die zum Teil deutliche Effekte der untersuchten Humankapitalfaktoren auf die Erfolgschancen neugegründeter Betriebe ergeben (statt vieler Boswell 1972; Klandt 1984; Picot et al. 1989), dann besagen die Befunde auch, daß Frauen mit tendenziell ungünstigeren Ausgangsvoraussetzungen den Schritt in die berufliche Selbständigkeit vollziehen.

Auf die Frage nach den Ursachen der geschlechtsspezifischen Unterschiede in der Ausstattung mit allgemeinem und unternehmerischem Humankapital soll an dieser Stelle nicht weiter eingegangen werden. Soweit es sich um die Unterschiede in der generellen Humankapitalausstattung (Bildung, Berufserfahrung) handelt, sei verwiesen auf die Ergebnisse der allgemeinen Forschung zur geschlechtsspezifischen Ungleichheit (z. B. Blau/Ferber 1986) und auch speziell auf die Ergebnisse der sozialwissenschaftlichen Frauenarbeitsforschung, in der vielfach Argumente einer frauenspezifischen Humankapitalausstattung bzw. eines ,weiblichen Arbeitsvermögens" vorgetragen werden (z.B. Beck-Gernsheim 1976; Ostner 1990). Die auch nachfrageseitigen Mechanismen, die den Frauen eine Aneignung unternehmerischen Humankapitals (Führungserfahrung, Selbständigkeitserfahrung) erschweren, dürften den Mechanismen im Bereich des Erwerbs von allgemeinem Humankapital sehr ähnlich sein. Kurz: Ohne die Bedeutung der Frage nach den Ursachen bestreiten zu wollen, beschränken wir uns hier auf eine Feststellung der bestehenden Unterschiede.

\section{Art der von Frauen und Männern gegrïndeten Betriebe}

Unabhängig von der Humankapitalausstattung soll im zweiten Schritt nun der Frage nachgegangen werden, ob und inwieweit sich Frauen und Männer hinsichtlich der Art der von ihnen gegründeten Betriebe unterscheiden. Ausgehend von der weithin bekannten Tatsache, daß Frauen auf dem allgemeinen Arbeitsmarkt eher auf die marginalen beruflichen Positionen (mit geringer Entlohnung, geringen Aufstiegschancen, geringen Entscheidungsbefugnissen usw.) verwiesen sind (dazu statt vieler Dahlke 1986; Engelbrech 1989, 1991), lautet dabei unsere erste Hypothese, daß sich Frauen auch im Bereich der beruflichen Selbständigkeit stärker in eher randständigen Selbständigkeitsexistenzen wiederfinden. Als randständige Selbständigkeitsexistenzen können dabei im Durchschnitt eher sehr kleine Betriebe, Betriebe mit geringem Startkapital, ohne abhängig Beschäftigte und Firmen in der Form von Kleingewerbebetrieben bezeichnet werden. Frauen dürften nicht zuletzt aufgrund ihrer geringeren Einkommenschancen in ab- 
hängigen Beschäftigungsverhältnissen in der Regel seltener in der Lage sein, das für eine erfolgversprechende Betriebsgründung erforderliche Startkapital aufzubringen. Weiterhin dürfte „Selbständigkeit aus der Ökonomie der Not" (Bögenhold 1987) bei Frauen relativ häufiger als Gründungsmotivation eine Rolle spielen als „Selbständigkeit aus der Ökonomie der Selbstverwirklichung“, oder anders formuliert, „push incentives“ im Unterschied zu „pull incentives" werden bei Frauen wichtiger sein als bei Männern.

Die Hauptergebnisse unserer Analysen zur Hypothese der relativ stärkeren Randständigkeit von weiblichen Betriebsgründungen sind in Tabelle 2 zusammengestellt. Wiederum ergibt sich eine überraschend deutliche Bestätigung der Ausgangserwartungen: Während Frauen im Durchschnitt $47000 \mathrm{DM}$ an Startkapital aufwenden, beläuft sich das arithmetische Mittel des aufgebrachten Startkapitals bei den Männern auf 166000 DM. Da die Verteilung des Startkapitals in hohem Maße rechtsschief ist (d.h. es gibt einige wenige Betriebsgründungen mit einem sehr hohen Startkapital), eignet sich der Medianwert des Startkapitals (5000 DM bei Frauen und 30000 DM bei Männern) besser zur Charakterisierung der Verteilung. $36 \%$ der Gründerinnen gegenüber $21 \%$ bei den Gründern geben an, daß sie überhaupt kein Startkapital im Zuge ihrer Betriebsgründung einsetzten. Auch bei der Zahl der Beschäftigten zum Zeitpunkt der Gründung, wobei die Gründerin bzw. der Gründer, sofern sie selbst in dem Betrieb gearbeitet haben, miteingerechnet sind (Halbtagskräfte sind mit 0.5 gezählt), gibt es eine signifikante Geschlechtsdifferenz. 63\% der von Frauen gegründeten Betriebe starteten als reine Ein-Personen-Firmen; bei den von Männern gegründeten Betrieben sind es nur $48 \%$. Frauen gründen mit $84 \%$ sehr viel häufiger Firmen mit der Rechtsform eines Kleingewerbebetriebes als Männer mit $63 \%$. Verwenden wir die drei Kriterien "Startkapital“, „Zahl der Beschäftigten zum Zeitpunkt der Gründung" und „Rechtsform" als einander ergänzende Maße der Startgröße einer betrieblichen Neugründung, können wir also eindeutig feststellen, daß Frauen in jeglicher Hinsicht kleinere Betriebe gründen.

91\% der von Frauen eröffneten Betriebe sind in den beiden Wirtschaftsbereichen „Handel“" und "Dienstleistungen“ angesiedelt. Demgegenüber sind die Bereiche „Industrie/verarbeitendes Gewerbe/Bau“ bei den Männern mit $17 \%$ deutlich stärker vertreten. Keinen signifikanten ge-
Tabelle 2 Art der von Frauen und Männern gegründeten Betriebe

\begin{tabular}{ll}
$\begin{array}{l}\text { Frauen } \\
(n=481)\end{array}$ & $\begin{array}{l}\text { Mănner } \\
(n=1368)\end{array}$ \\
\hline
\end{tabular}

Startkapital in DM**

Arith. Mittel

47000

5000

166000

Median

2.16

30000

Zahl der Beschäftigten

zum Zeitpunkt der Gründung *

Rechtsform ${ }^{* *}$

Kleingewerbe

Handelsregisterfirma

$84 \%$

$16 \%$

$63 \%$

$37 \%$

Wirtschaftsbereich ${ }^{\star *}$

Industrie/Bau

Handel

Dienstleistung

$\begin{array}{rr}9 \% & 17 \% \\ 40 \% & 36 \% \\ 51 \% & 47 \%\end{array}$

Gründungsart 1

Neugründung

Firmenübernahme

$74 \%$

$26 \%$

$76 \%$

$24 \%$

Gründungsart 2**

Alleingründung

Partnergründung

$80 \%$

$20 \%$

$68 \%$

$32 \%$

Gründungsintention $1^{* \star}$

Nebenenwerbsgründung

Vollerwerbsgründung

$41 \%$

$59 \%$

$29 \%$

$71 \%$

Gründungsintention 2**

Erzielung eines

ausreichenden Einkommens $72 \%$

$61 \%$

Erzielung eines

möglichst hohen Gewinns $\quad 28 \%$

$39 \%$

Gründungsintention $3^{* *}$

Bedienung ausschließlich

des lokalen Marktes

Bedienung auch des

überregionalen Marktes

$66 \%$

$52 \%$

$34 \%$

$48 \%$

"* Unterschied zwischen den Subgruppen (Frauen-Mănner) ist signifikant auf dem $1 \%$-Niveau; * signifikant auf dem 5\%-Niveau; aufgrund fehlender Werte bei den Aufgliederungsmerkmalen vermindern sich in den beiden Subgruppen zum Teil die Fallzahlen geringfügig.

schlechtsspezifischen Unterschied beobachten wir bei dem Merkmal, ob es sich bei der Gründung um einen vollständig neuen Betrieb handelte oder um eine Firmenübernahme. Jeweils rund ein Viertel der Männer und Frauen machen sich selbständig, indem sie einen zuvor bereits bestehenden Betrieb (z. B. den elterlichen Betrieb oder den Betrieb des Ehepartners) übernehmen. Mit $80 \%$ - gegenüber $68 \%$ bei den Männern - gründen Frauen ihren 
Betrieb überdurchschnittlich häufig allein, d.h. ohne eine(n) Geschäftspartner(in). Weiterhin gründen Frauen ihren Betrieb signifikant häufiger mit der Intention eines Nebenerwerbs. Für $41 \%$ der Frauen hat die Betriebsgründung lediglich die Funktion eines Nebenerwerbs; bei den Männern beläuft sich der entsprechende Prozentsatz auf $29 \%$. Dies deutet darauf hin, daß Frauen in der Tat sehr häufig ihre Selbständigkeit von Anfang an als (finanzielle) Ergänzung zur Familien- bzw. Kindererziehungsarbeit konzipieren. Während von den Frauen in unserer Studie, die keine Kinder hatten, $31 \%$ eine Nebenerwerbsgründung unternahmen, waren es bei Frauen mit Kindern $44 \%$. Die Unterschiede auf den Dimensionen „Alleinversus Partnergründung“ und "Neben- versus Vollerwerbsgründung" können als weiterer Beleg dafür gelten, daß Frauen tendenziell kleinere und damit eher randständige Selbständigkeitsexistenzen begründen.

Auch von ihrem individuellen Anspruchsniveau her und von den mit der Betriebsgründung verbundenen Ambitionen geben sich die Frauen in der Regel deutlich bescheidener als die Männer. Für 72\% der Frauen ist die Erzielung eines ausreichenden Einkommens, also die bloße Subsistenzsicherung, der Hauptzweck der Betriebsgründung; bei den Männern geben sich nur $61 \%$ damit zufrieden. Umgekehrt deklarieren Männer deutlich häufiger als Frauen die Erzielung eines möglichst hohen Gewinns als wichtiges Anliegen ihrer Betriebsgründung. Das geringere Anspruchsniveau der Frauenbetriebe drückt sich auch in der Reichweite des angezielten Marktes aus. Frauen beschränken sich mit $66 \%$ eher als Männer (52\%) darauf, mit ihrem Betrieb ausschließlich den lokalen Markt zu bedienen. Mithin läßt sich festhalten, daß Frauenbetriebe überdurchschnittlich häufig als "Locals“ agieren.

Bereits in Tabelle 2 haben wir die Verteilung der Frauen- und Männerbetriebe auf grobe Wirtschaftsbereiche (Industrie/Bau, Handel, Dienstleistungen) betrachtet. Diese Analyse soll im folgenden noch etwas vertieft werden. Ausgangspunkt hierbei waren für uns die zwei in der allgemeinen Arbeitsmarktforschung diskutierten Phänomene, daß es erstens vielfach eine geschlechtsspezifische Segregation der Berufe bzw. Branchen in ,frauen-“ und "männertypisch" gibt und $\mathrm{daB}$ sich zweitens häufig eine relativ stärkere Konzentration der Frauen auf bestimmte Berufe bzw. Branchen beobachten läßt (vgl. dazu statt vieler Diekmann 1985; Willms-Herget 1985; Blossfeld 1987; RabeKleberg 1987). Übertragen auf den Bereich der beruflichen Selbständigkeit lautete demnach die Arbeitshypothese unserer Analysen, da $\beta$ Frauen eher Betriebe in "frauentypischen" Branchen gründen und sich dabei stärker auf einige wenige Branchen konzentrieren.

Die genannte Hypothese wollen wir überprüfen, indem wir die zehn am stärksten besetzten Branchen bei den Frauen und Männern einander gegenüberstellen. Tabelle 3 zeigt das Ergebnis dieser Gegenüberstellung. Bezogen auf die Teilthese einer stärkeren Konzentration der Frauen auf bestimmte Branchen erhalten wir eine, wenngleich insgesamt eher schwache Bestätigung. Die fünf am stärksten von den Frauen besetzten Branchen dekken $34 \%$ aller Frauenbetriebe ab, die fünf am stärksten von den Männern besetzten Branchen 26\% der Männerbetriebe. Zieht man die zehn am stärksten besetzten Branchen heran, ergibt sich

Tabelle 3 Die zehn häufigsten Branchen bei Frauen und Männern.

\begin{tabular}{lcccccl}
\hline Frauenbranchen & N & $\%$ & Rang & $\%$ & N & Männerbranchen \\
\hline Handel mit Textilien & 38 & 7.9 & 1 & 5.9 & 81 & EDV-Dienstleistungen \\
Schreibbüros & 35 & 7.3 & 2 & 5.6 & 77 & Beratung von Unternehmen \\
Gaststätten & 33 & 6.9 & 3 & 5.5 & 75 & Verkehr/Spedition \\
Beratung von Unternehmen & 31 & 6.4 & 4 & 4.5 & 62 & Versicherung \\
Handel mit Nahrungsmittein & 27 & 5.6 & 5 & 4.5 & 62 & Gaststätten \\
Handel mit Kosmetikartikeln & 23 & 4.8 & 6 & 4.0 & 54 & Herstellung von elektrotechnischen Produkten \\
Herstellung von Textilien & 17 & 3.5 & 7 & 4.0 & 54 & Handel mit Nahrungsmitteln \\
Versicherung & 15 & 3.1 & 8 & 3.6 & 49 & Handel mit Textilien \\
Handel mit Spielwaren & 14 & 2.9 & 9 & 3.4 & 46 & Druckgewerbe \\
Handel mit Schreibwaren & 14 & 2.9 & 10 & 3.0 & 41 & Handel mit KfZ(-Artikeln) \\
\hline Insgesamt: Frauen & 247 & 51.3 & & 44.0 & 604 & Männer: Insgesamt \\
\hline
\end{tabular}


eine kumulierte Häufigkeit von $51 \%$ bei den Frauen und $44 \%$ bei den Männern. Überblicken wir dann zunächst die "Hitliste" der Frauenbetriebe im einzelnen, gelangt man in der Tat zu dem Gesamtbild, daß im herkömmlichen Sinne als „frauennah“ bezeichnete Branchen von den Frauen überdurchschnittlich häufig gewählt werden. Zweimal taucht der Bereich Textilien auf (Handel und Herstellung von Textilien), an zweiter Stelle stehen die Schreibbüros, weiterhin begegnen wir dem Handel mit Nahrungsmitteln, Kosmetikartikeln sowie Spiel- und Schreibwaren. Demgegenüber stehen in der Männerliste fünf Branchen, die in der Frauenliste überhaupt nicht auftauchen, nämlich EDV-Dienstleistungen (als Rang 1 bei den Männern), Verkehr/Spedition, Herstellung von elektrotechnischen Produkten, Druckgewerbe und Handel mit KfZ bzw. KfZ-Artikeln. Insgesamt beobachten wir damit Unterschiede der Branchenwahl, die weitgehend dem Stereotyp von frauen- bzw. männerspezifischen Tätigkeitsbereichen folgen.

Wiederum können und sollten jedoch die in diesem Abschnitt aufgezeigten Unterschiede in der Art der von Frauen und Männern gegründeten Betriebe nicht direkt als Beleg dafür herangezogen werden, daß berufliche Selbständigkeit für Frauen keine erfolgversprechende Alternative ist bzw. daß Frauen die schlechteren Unternehmer sind. Ähnlich wie bei der Humankapitalausstattung läßt sich jedoch immerhin argumentieren, daß Frauen aufgrund der Art der Betriebe, die sie gründen (kleine Betriebe, Nebenerwerbsbetriebe, wenig kapitalintensive Betriebe im Handel und Dienstleistungssektor), tendenziell die schlechteren Ausgangschancen haben.

Vor allem unser Verweis auf das geringere Anspruchsniveau und die begrenzte Ambitioniertheit der Frauenbetriebe sollte nicht in dem Sinne mißverstanden werden, daß wir hierbei von fixen, geschlechtsspezifischen Dispositionen ausgehen. Hält man sich vor Augen, daß Frauen vielfach gleichzeitig noch die Familienarbeit zu erledigen haben, daß sie nur mit einem geringen Startkapital in die Selbständigkeit eintreten können und daß sie häufiger eher Quereinsteigerinnen sind, erscheint eine Reaktion nach dem Motto „Klein, aber fein“ als durchaus rational.

\section{Bestands- und Überlebenschancen von Frauen- und Männerbetrieben}

Nachdem wir in den beiden vorangehenden $\mathrm{Ab}$ schnitten festgestellt haben, daß Frauen zum einen mit einer ungünstigeren Humankapitalausstattung in die berufliche Selbständigkeit eintreten und zum anderen Betriebe gründen, die tendenziell eher als randständige Selbständigkeitsexistenzen gesehen werden müssen, kommen wir nun zu dem - mit Blick auf die Fragestellung der Arbeit - entscheidenden Untersuchungsschritt, der die relativen Erfolgschancen von Frauen- und Männerbetrieben vergleicht.

Betrieblicher Erfolg soll dabei zunächst an den Bestands- und Überlebenschancen der Betriebe festgemacht werden. Obwohl die Beendigung eines Betriebes nicht ohne weiteres mit Mißerfolg gleichgesetzt werden darf (im günstigen Fall kann ein Betrieb z. B. gewinnbringend verkauft werden), läßt sich auf der Basis unserer Daten immerhin belegen, daß eine hohe Koinzidenz besteht. Mindestens zwei Drittel aller Betriebsauflösungen in unserer Studie können, gemessen an verschiedenen Kriterien (v. a. am Kriterium finanzieller Verluste), gleichzeitig als „betriebliches Scheitern“ qualifiziert werden. Bestand bzw. Überleben kann zudem sicherlich als Minimalkriterium betrieblichen Erfolges gelten.

Als statistische Analyseverfahren zur Untersuchung der Überlebenschancen der Betriebe verwenden wir Methoden der Ereignisdatenanalyse (dazu z. B. Diekmann/Mitter 1984; Blossfeld et al. 1986). Mittels Sterbetafel sollen zunächst die jeweiligen Überlebensquoten der Betriebe betrachtet werden. Im Rahmen der multivariaten Analyse werden anschließend mit dem semi-parametrischen Modell der Cox-Regression die Effekte verschiedener Determinanten der betrieblichen „Sterberate" untersucht.

Die empirischen Befunde der Abschnitte 3 und 4 führen im ersten Schritt fast zwangsläufig auf die Erwartung, daß die von Frauen gegründeten Betriebe insgesamt schlechtere Überlebenschancen haben als die Männerbetriebe. Daß dies tatsächlich der Fall ist, geht aus den Überlebensfunktionen in Abbildung 1 hervor. Beginnend vom Zeitpunkt der Gründung geben die mit der Sterbetafelmethode berechneten Überlebensfunktionen den Anteil der zu jedem Zeitpunkt noch bestehenden Betriebe an. Aus den Überlebensfunktionen in Abbildung 1 läßt sich unter anderem ablesen, daß zwei Jahre nach der Gründung nur noch $72 \%$ der 


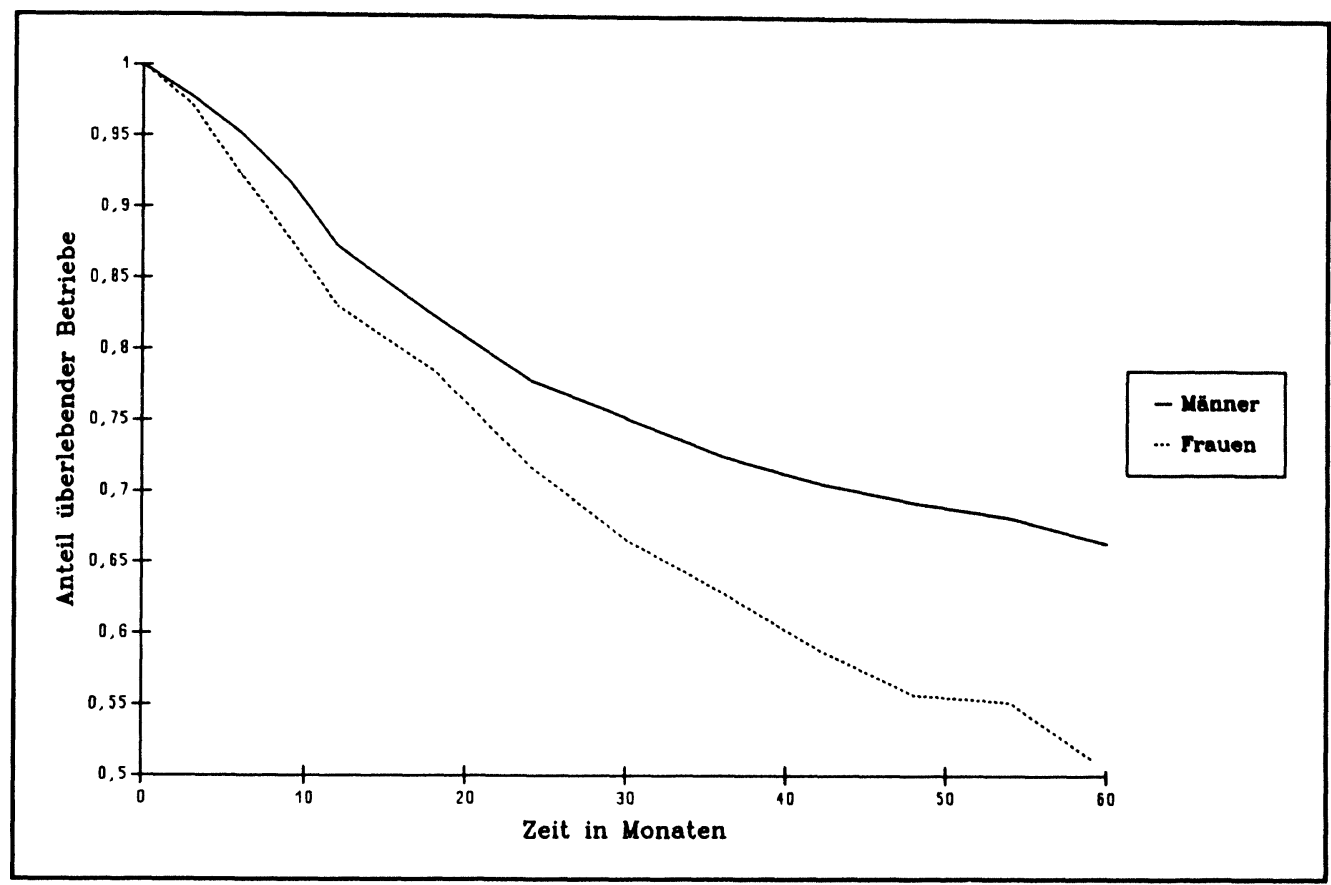

Abbildung 1 Überlebensfunktionen der von Frauen und Männern gegründeten Betriebe.

Frauenbetriebe, aber noch $78 \%$ der Männerbetriebe bestanden. Nach fünf Jahren sind $50 \%$ der Frauenbetriebe gegenüber $66 \%$ der Männerbetriebe noch existent.

Die zentrale Anschlußfrage nun aber ist, ob die von Frauen gegründeten Betriebe dieselben Bestandschancen hätten, wenn die Gründerinnen mit der gleichen Humankapitalausstattung wie die Gründer in die Selbständigkeit einträten und/oder wenn die Frauen dieselbe Art von Betrieben gründeten wie die Männer. Diese Frage kann mittels multivariater Analyse untersucht werden, wobei neben dem Geschlecht - zum einen Aspekte der Humankapitalausstattung der Gründer/innen und zum anderen Basismerkmale der gegründeten Betriebe kontrolliert werden müssen. Für die multivariate Analyse verwenden wir, wie schon erwähnt, die Cox-Regression, bei der die Sterberate der Betriebe als abhängige Variable dient. Die über die Cox-Regression geschätzten Parameter lassen sich sehr anschaulich im Sinne von Prozenteffekten auf die Sterberate interpretieren, wobei Parameterwerte größer als Eins einen positiven Effekt der jeweiligen Variable auf die Sterberate, Werte kleiner als Eins hingegen einen negativen Effekt bedeuten.
Die Ergebnisse unserer multivariaten Modellschätzungen mit der Cox-Regression finden sich in Tabelle 4. Im ersten Modell von Tabelle 4 (Modell 1) wird zunächst ein Basismodell geschätzt, das lediglich Geschlecht als Einflußgröße auf die Sterberate der Betriebe enthält. Erwartungsgemäß zeigt sich, $\mathrm{da} ß$ die von Frauen gegründeten Betriebe eine signifikant höhere (genauer: eine um 53\% höhere) Sterberate haben als die Männerbetriebe. Modell 2 kontrolliert zusätzlich für die Humankapitalausstattung der Gründer/innen, Modell 3 für einige zentrale Basismerkmale der Betriebe und Modell 4 schließlich sowohl für die Humankapitalausstattung als auch für die Betriebscharakteristika. In allen drei Modellen mit zusätzlichen Kovariaten verschwindet die Signifikanz des Geschlechtseffekts, wobei (allerdings nicht signifikant) in Modell 4 die von Frauen gegründeten Betriebe sogar eine um 5\% geringere Sterberate haben als die Männerbetriebe. Mithin gelangen wir zu der eindeutigen Schlußfolgerung: Wenn Frauen mit derselben $\mathrm{Hu}$ mankapitalausstattung in die berufliche Selbständigkeit einträten wie die Männer und/oder wenn Frauen dieselbe Art von Betrieben gründeten, gäbe es keine geschlechtsspezifischen Unterschiede der Bestandschancen von Männer- und Frauenbetrieben. 
Tabelle 4 Determinanten der Sterberate neugegründeter Betriebe (Cox-Regression, Alpha-Koeffizienten).

\begin{tabular}{|c|c|c|c|c|}
\hline Kovariate & Modell 1 & Modell 2 & Modell 3 & Modell 4 \\
\hline $\begin{array}{l}\text { Geschlecht } \\
(1=\text { Frau })\end{array}$ & $\begin{array}{l}1.53^{\star \star} \\
(4.98)\end{array}$ & $\begin{array}{l}1.10 \\
(1.03)\end{array}$ & $\begin{array}{l}1.04 \\
(0.45)\end{array}$ & $\begin{array}{l}0.95 \\
(0.57)\end{array}$ \\
\hline Bildung in Jahren & & $\begin{array}{l}0.91^{* *} \\
(5.58)\end{array}$ & & $\begin{array}{l}0.94^{\star *} \\
(3.72)\end{array}$ \\
\hline Berufserfahrung in Jahren & & $\begin{array}{l}0.97^{\star *} \\
(5.30)\end{array}$ & & $\begin{array}{l}0.98^{* \star} \\
(3.77)\end{array}$ \\
\hline $\begin{array}{l}\text { Branchenerfahrung } \\
(1=\mathrm{Ja})\end{array}$ & & $\begin{array}{l}0.46^{\star *} \\
(8.89)\end{array}$ & & $\begin{array}{l}1.56^{* *} \\
(4.87)\end{array}$ \\
\hline $\begin{array}{l}\text { Selbständigkeitserfahrung } \\
(1=\mathrm{Ja})\end{array}$ & & $\begin{array}{l}0.86 \\
(1.46)\end{array}$ & & $\begin{array}{l}0.95 \\
(0.51)\end{array}$ \\
\hline $\begin{array}{l}\text { Startkapital in DM } \\
\text { (logarithmiert) }\end{array}$ & & & $\begin{array}{l}0.97^{* *} \\
(3.76)\end{array}$ & $\begin{array}{l}0.97^{* *} \\
(3.42)\end{array}$ \\
\hline $\begin{array}{l}\text { Beschäftigte bei Gründung } \\
\text { (logarithmiert) }\end{array}$ & & & $\begin{array}{l}0.75^{\star *} \\
(4.57)\end{array}$ & $\begin{array}{l}0.76^{* \star} \\
(3.98)\end{array}$ \\
\hline $\begin{array}{l}\text { Rechtsform } \\
\text { ( } 1 \text { = Handelsregisterfirma) }\end{array}$ & & & $\begin{array}{l}0.31^{\star \star} \\
(8.01)\end{array}$ & $\begin{array}{l}0.37^{* *} \\
(6.45)\end{array}$ \\
\hline $\begin{array}{l}\text { Voll- oder Nebenerwerbsbetrieb } \\
(1 \text { = Vollerwerbsgründung) }\end{array}$ & & & $\begin{array}{l}0.79^{*} \\
(2.53)\end{array}$ & $\begin{array}{l}0.86 \\
(1.55)\end{array}$ \\
\hline Branche: Handel & & & $\begin{array}{l}1.77^{\star \star} \\
(3.63)\end{array}$ & $\begin{array}{l}1.63^{\star \star} \\
(3.02)\end{array}$ \\
\hline Branche: Dienstleistung & & & $\begin{array}{l}1.54^{\star *} \\
(2.78)\end{array}$ & $\begin{array}{l}1.47^{\star} \\
(2.43)\end{array}$ \\
\hline $\begin{array}{l}\text { Chi-Quadrat (di) } \\
\text { Anteil Zensierte } \\
\mathbf{N}\end{array}$ & $\begin{array}{c}25.2(1) \\
65.5 \\
1793\end{array}$ & $\begin{array}{c}216.8(5) \\
65.9 \\
1760\end{array}$ & $\begin{array}{l}298.6(7) \\
65.3 \\
1777\end{array}$ & $\begin{array}{c}357.7(11) \\
65.7 \\
1745\end{array}$ \\
\hline
\end{tabular}

"Signifikant auf dem 1\%-Niveau; " signifikant auf dem 5\%-Niveau; t-Werte in Klammern. Basiskategorien: Männer; keine Branchenerfahrung; keine Selbständigkeitserfahrung; Kleingewerbebetrieb; Nebenerwerbsgründung; Industrie/Baugewerbe.

Bezogen auf die Forschungsfrage der vorliegenden Arbeit bedeutet dies, daß unter dem Gesichtspunkt der Bestandsfestigkeit ihrer Betriebe Frauen sicherlich nicht als die schlechteren Unternehmer eingestuft werden können. Während im Bereich der abhängigen Beschäftigung signifikante Geschlechtsunterschiede (z. B. im Rahmen von Einkommensfunktionen oder in Modellen innerbetrieblicher Aufstiegschancen) in der Regel auch bei Kontrolle von Humankapitalfaktoren, betrieblichen Kontextmerkmalen usw. sehr wohl erhalten bleiben, gilt dies nicht im Bereich der beruflichen Selbständigkeit hinsichtlich des Erfolgskriteriums der Bestandsfähigkeit der Betriebe. Diesen Befund werten wir als ein Indiz zugunsten der Selbständigkeitsoption für Frauen.

Obwohl uns in den Modellen von Tabelle 4 vor allem der Geschlechtseffekt interessierte, sei ab- schließend zu diesem Abschnitt noch kurz ein Blick auf die anderen, in den Modellen berücksich tigten Bestimmungsfaktoren der Überlebenschancen der Betriebe geworfen: Die allgemeinen $\mathrm{Hu}$ mankapitalressourcen der Gründer/innen, erfaßt über die Jahre der Bildung (schulische und berufliche Bildung) und über die Jahre der Berufserfahrung, zeigen einen deutlichen Effekt dergestalt, $\mathrm{da} B$ sie die Sterberate der Betriebe verringern bzw. die Überlebenschancen erhöhen. Unter den zwei berücksichtigten Aspekten des unternehmerischen Humankapitals erweist sich vorherige Branchenerfahrung als von zentraler Bedeutung, während vorherige Selbständigkeitserfahrung keinen signifikanten Einfluß ergibt. Für die vier Indikatoren der Startgröße einer Betriebsgründung (Startkapital, Zahl der Beschäftigten zum Zeitpunkt der Gründung, Rechtsform und Voll- bzw. Nebenerwerbs- 
gründung) erhalten wir sehr deutliche Effekte in der Form, daß kleinere Betriebe einem erhöhten Sterberisiko ausgesetzt sind. Weiterhin sind - im Vergleich zu Betrieben des verarbeitenden Gewerbes (Industrie) und im Bausektor - Betriebe im Handel und Dienstleistungsfirmen (auch bei Kontrolle der Gründungsgröße) stärker vom Risiko des Scheiterns betroffen.

\section{Beschäftigten- und Umsatzentwicklung der Frauen- und Männerbetriebe}

Für die Betriebe, die den in unserer Studie gewählten Beobachtungszeitraum (1985/86 bis Anfang 1990) überleben konnten, also für die "Survivor“Einheiten, soll im weiteren noch untersucht werden, wie sich diese Betriebe im Zeitablauf entwikkelten, wobei wiederum die geschlechtsspezifischen Unterschiede im Mittelpunkt des Interesses stehen. Als Größen zur Charakterisierung der Betriebsentwicklung verwenden wir zum einen die Entwicklung der Zahl der Beschäftigten (unter Einschluß der Gründungsperson) und zum anderen die ebenfalls auf Jahresbasis erhobene Umsatzentwicklung.
Der Befund, daß Frauen im Durchschnitt kleinere Betriebe gründen, läßt dabei zunächst die Vermutung entstehen, daß Frauenbetriebe eher einem „Expansionskurs“ folgen als Männerbetriebe, da es bei einem niedrigeren Ausgangsniveau leichter sein dürfte, mehr oder weniger stark ,zuzulegen“. Abbildung 2 (Beschäftigtenentwicklung) und $\mathrm{Ab}$ bildung 3 (Umsatzentwicklung) zeigen jedoch, daß dies nicht ohne weiteres der Fall ist.

Die durchschnittliche Zahl der Beschäftigten der von Männern gegründeten (und durchgehend bestehenden) Betriebe beträgt im ersten Betriebsjahr 3.86 und im fünften Betriebsjahr 5.83, ein $\mathrm{Zu}$ wachs von 1.97 Beschäftigten bzw. eine prozentuale Steigerung von $51 \%$. Die entsprechenden Werte bei den Frauenbetrieben sind 3.02 im ersten Jahr, 3.96 im fünften Jahr, ein Zuwachs von $0.94 \mathrm{Be}-$ schäftigten und eine prozentuale Steigerung von $31 \%$. Mithin sind sowohl die absoluten als auch die relativen durchschnittlichen Zuwächse bei den Männerbetrieben größer als bei den Frauenbetrieben. Die Werte bei der Umsatzentwicklung gestalten sich wie folgt: Die Männerbetriebe starten mit einem durchschnittlichen Umsatz im ersten Geschäftsjahr von $906000 \mathrm{DM}$, im vierten abgeschlos-

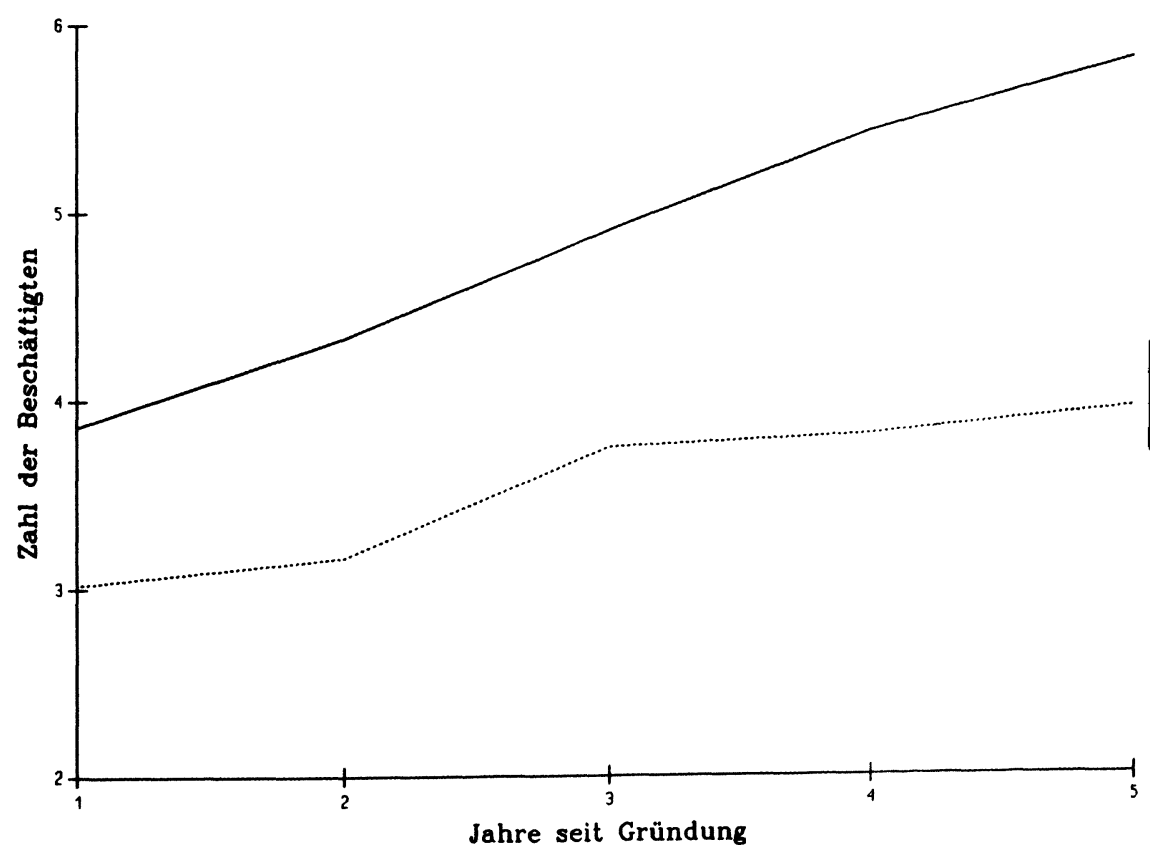

Abblldung 2 Beschäftigtenentwicklung der von Frauen und Männern gegründeten Betriebe (Survivor-Einheiten). 


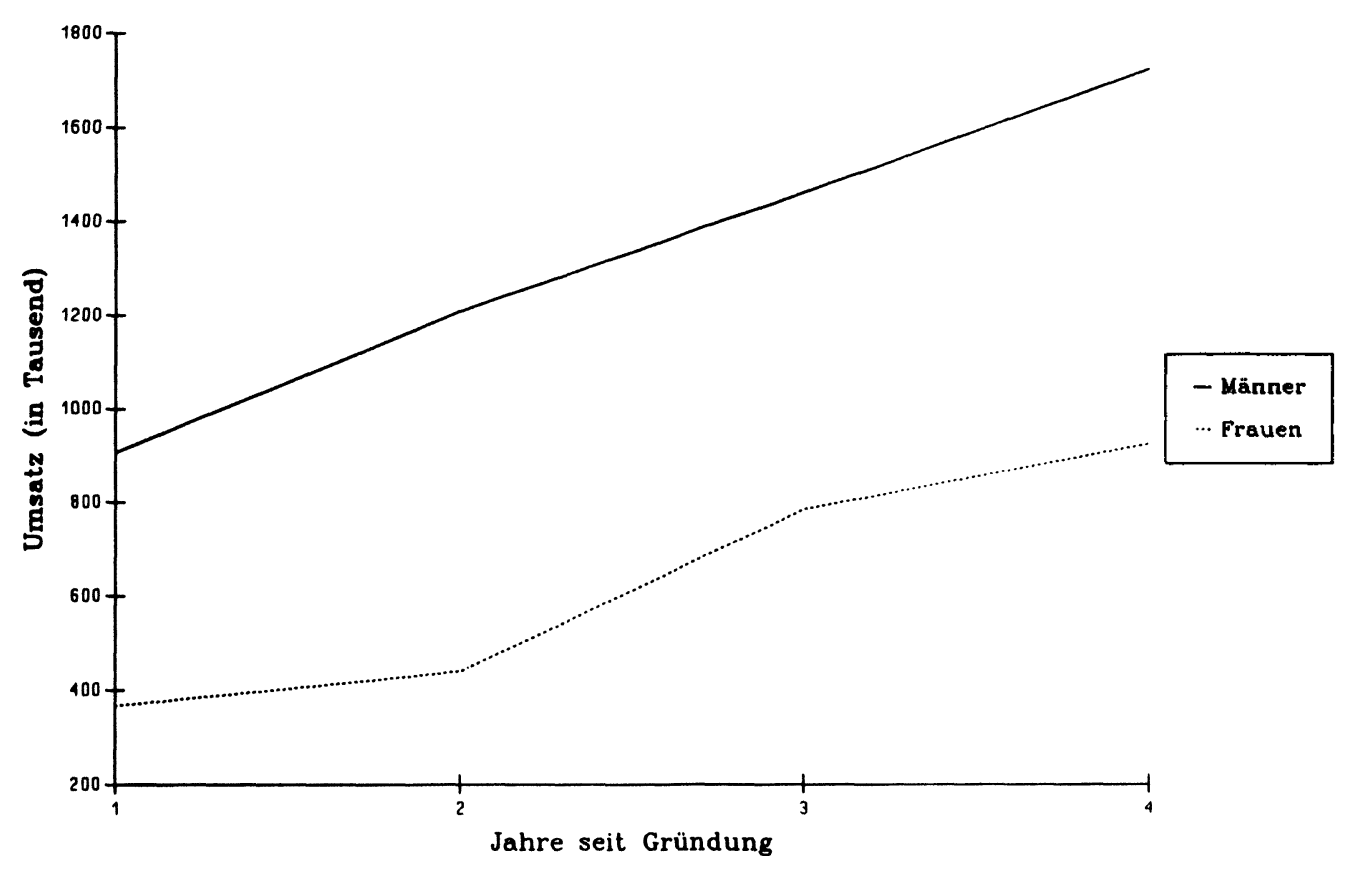

Abblldung 3 Umsatzentwicklung der von Männern und Frauen gegründeten Betriebe (Survivor-Einheiten).

senen Geschäftsjahr liegt der Umsatz bei durchschnittlich 1726000 DM, eine absolute Steigerung von 820000 DM und eine prozentuale von $91 \%$. Für die Frauenbetriebe belaufen sich die Umsatzwerte auf $367000 \mathrm{DM}$ im ersten Geschäftsjahr und $926000 \mathrm{DM}$ im vierten Geschäftsjahr, was eine absolute Steigerung von $559000 \mathrm{DM}$ und eine relative von $152 \%$ bedeutet. Zunächst analog zur Beschäftigtenentwicklung ist also zwar der absolute durchschnittliche Umsatzzuwachs bei den Männerbetrieben höher als bei den Frauenbetrieben, nicht jedoch der relative. Beim relativen Umsatzzuwachs dominiert offenbar der Effekt des unterschiedlichen Ausgangsniveaus, das die Möglichkeiten von Umsatzsteigerungen begrenzt.

Wie schon bei der Analyse der Überlebenschancen der Neugründungen soll im nächsten Schritt nun wiederum untersucht werden, ob die Geschlechtsunterschiede bei der Beschäftigten- und Umsatzentwicklung bestehen bleiben, wenn man zusätzlich für die Humankapitalausstattung der Gründer/ innen und für die Unterschiede bei der Art der gegründeten Betriebe kontrolliert. Als abhängige Variable für diese multivariaten Analysen wollen wir dabei lediglich die Information verwenden, ob ein Betrieb vom ersten bis zum fünften Jahr seine Beschäftigtenzahl erhöht hat oder nicht bzw. ob vom ersten bis zum vierten (vollen) Geschäftsjahr eine Steigerung des Jahresumsatzes zu verzeichnen ist oder nicht. Eine Steigerung wird jeweils mit 1, Konstanz bzw. eine Abnahme jeweils mit 0 verschlüsselt. ${ }^{5}$ Als statistisches Auswertungsverfahren bietet sich bei der vorgeschlagenen $0 / 1$-Vercodung der abhängigen Variablen die logistische Regression an (vgl. dazu z. B. Aldrich/Nelson 1984).

Die Ergebnisse der verschiedenen logistischen Regressionsmodelle, bei denen der gleiche Kovariatensatz wie bei den Cox-Regressionen verwendet wird, finden sich in Tabelle 5. In der Ausgangssi-

${ }^{5}$ Diese Vereinfachung der Ausgangsinformation wurde vor allem deshalb vorgenommen, um die enorme Variabilität der individuellen betrieblichen Entwicklungen in den Griff zu bekommen. Eine detailliertere Analyse könnte die relativen (oder auch absoluten) jährlichen Steigerungen der Beschäftigtenzahl bzw. des Umsatzes untersuchen. Wollte man zudem noch die abgemeldeten Betriebe in die Analyse einbeziehen, ergäben sich eine Reihe zusätzlicher methodischer Probleme. 
Tabelle 5 Determinanten des Beschäftigten- und Umsatzzuwachses neugegründeter Betriebe (Logistische Regression).

\begin{tabular}{|c|c|c|c|c|}
\hline \multirow[b]{2}{*}{ Kovariate } & \multicolumn{2}{|c|}{$\begin{array}{l}\text { Determinanten der } \\
\text { Beschäftigtenentwicklung }\end{array}$} & \multicolumn{2}{|c|}{$\begin{array}{l}\text { Determinanten der } \\
\text { Umsatzentwicklung }\end{array}$} \\
\hline & Modell 1 & Modell 2 & Modell 1 & Modell 2 \\
\hline $\begin{array}{l}\text { Geschlecht } \\
(1=\text { Frau })\end{array}$ & $\begin{array}{r}-0.92^{\star \star} \\
(5.57)\end{array}$ & $\begin{array}{r}-0.75^{* *} \\
(4.07)\end{array}$ & $\begin{array}{r}-0.45^{\star} \\
(2.46)\end{array}$ & $\begin{array}{c}-0.32 \\
(1.56)\end{array}$ \\
\hline Bildung in Jahren & & $\begin{array}{l}0.02 \\
(0.85)\end{array}$ & & $\begin{array}{l}-0.03 \\
(0.88)\end{array}$ \\
\hline Berufserfahrung in Jahren & & $\begin{array}{r}-0.04^{* *} \\
(4.98)\end{array}$ & & $\begin{array}{r}-0.03^{* *} \\
(2.90)\end{array}$ \\
\hline $\begin{array}{l}\text { Branchenerfahrung } \\
(1=\mathrm{Ja})\end{array}$ & & $\begin{array}{l}0.38^{*} \\
(2.28)\end{array}$ & & $\begin{array}{l}0.57^{* *} \\
(2.97)\end{array}$ \\
\hline $\begin{array}{l}\text { Selbständigkeitserfahrung } \\
(1=\mathrm{Ja})\end{array}$ & & $\begin{array}{l}-0.24 \\
(1.59)\end{array}$ & & $\begin{array}{l}-0.32 \\
(1.82)\end{array}$ \\
\hline $\begin{array}{l}\text { Startkapital in DM } \\
\text { (logarithmiert) }\end{array}$ & & $\begin{array}{l}0.05^{* *} \\
(2.89)\end{array}$ & & $\begin{array}{l}0.06^{* *} \\
(2.67)\end{array}$ \\
\hline $\begin{array}{l}\text { Beschäftigte bei Gründung } \\
\text { (logarithmiert) }\end{array}$ & & $\begin{array}{l}-0.05 \\
(0.57)\end{array}$ & & $\begin{array}{l}-0.17 \\
(1.62)\end{array}$ \\
\hline $\begin{array}{l}\text { Rechtsform } \\
(1=\text { Handelsregisterfirma) }\end{array}$ & & $\begin{array}{l}0.73^{* *} \\
(4.48)\end{array}$ & & $\begin{array}{l}0.05 \\
(0.23)\end{array}$ \\
\hline $\begin{array}{l}\text { Voll- oder Nebenerwerbsbetrieb } \\
(1=\text { Vollerwerbsgründung) }\end{array}$ & & $\begin{array}{l}0.05 \\
(0.25)\end{array}$ & & $\begin{array}{l}0.71^{\star \star *} \\
(3.38)\end{array}$ \\
\hline Branche: Handel & & $\begin{array}{r}-0.46^{*} \\
(2.39)\end{array}$ & & $\begin{array}{l}-0.16 \\
(0.64)\end{array}$ \\
\hline Branche: Dienstleistung & & $\begin{array}{r}-0.41^{*} \\
(2.18)\end{array}$ & & $\begin{aligned}-0.48^{*} \\
(2.01)\end{aligned}$ \\
\hline Konstante & $\begin{array}{l}-0.07 \\
(0.95)\end{array}$ & $\begin{array}{l}-0.48 \\
(1.01)\end{array}$ & $\begin{array}{l}1.19^{* *} \\
(13.26)\end{array}$ & $\begin{array}{l}1.04 \\
(1.82)\end{array}$ \\
\hline $\begin{array}{l}\text { Pseudo- } R^{2} \\
N\end{array}$ & $\begin{array}{l}2.3 \% \\
1041\end{array}$ & $\begin{array}{l}9.2 \% \\
1015\end{array}$ & $\begin{array}{l}0.6 \% \\
870\end{array}$ & $\begin{array}{l}6.4 \% \\
853\end{array}$ \\
\hline
\end{tabular}

** Signifikant auf dem 1\%-Niveau; * signifikant auf dem 5\%-Niveau; t-Werte in Klammern. Basiskategorien: Männer; keine Branchenerfahrung; keine Selbständigkeitserfahrung; Kleingewerbebetrieb; Nebenerwerbsgründung; Industrie/Baugewerbe.

tuation verhält es sich dabei zunächst so, daß $39 \%$ der Männerbetriebe gegenüber $20 \%$ bei den Frauenbetrieben ihre Beschäftigtenzahl erhöhen und $66 \%$ der Männerbetriebe gegenüber $58 \%$ bei den Frauenbetrieben ihren Umsatz steigern konnten.

Berücksichtigt man in einem Basismodell (jeweils Modell 1 in Tabelle 5) zunächst ausschlieBlich die Geschlechtsvariable als Einflußfaktor, ergibt sich sowohl bei der Beschäftigten- als auch bei der Umsatzentwicklung ein signifikant negativer Effekt dergestalt, daß Frauenbetriebe seltener ihre Beschäftigtenzahl aufstocken und ihren Umsatz steigern. Auch bei zusätzlicher Kontrolle der Fak- toren der Humankapitalausstattung und der Basismerkmale der Betriebe (Modelle 2) bleibt im Fall der Beschäftigtenentwicklung der signifikante Geschlechtseffekt erhalten. Im Fall der Umsatzentwicklung bleibt der Geschlechtseffekt zwar negativ, unterscheidet sich jedoch nicht mehr signifikant für Männer und Frauen. Anders als bei den Bestands- und Überlebenschancen der Betriebe können wir damit schlußfolgern, daß Frauenbetriebe seltener ihren Mitarbeiterstab aufstocken als Männerbetriebe. Obwohl also von Frauen gegründete Betriebe - bei gleicher Humankapitalausstattung und bei einer gleichen Art von Gründung dieselben Überlebenschancen haben wie Männer- 
betriebe und auch tendenziell genauso häufig ein Umsatzplus erzielen, erweisen sich die Frauenbetriebe bei der Aufstockung der Beschäftigtenzahl als weniger expansiv. Frauen scheinen ihren Betrieb eher durch Konsolidierung als durch Expansion abzusichern. ${ }^{6}$

Wiederum freilich sollten uns diese Befunde nicht vorschnell dazu verleiten, Frauen pauschal als die schlechteren Unternehmer zu etikettieren bzw. die Selbständigkeitsoption für Frauen als wenig attraktiv auszuschließen. Im Gegenteil könnte man sogar argumentieren, daß die Tatsache, daß Frauenbetriebe trotz geringerer Beschäftigungsexpansion genauso bestandsfest sind wie die Männerbetriebe und ihren Umsatz ähnlich häufig steigern können, eigentlich für die unternehmerischen Qualitäten der Frauen spricht. ${ }^{7}$ Unter dem Aspekt der ökonomischen Folgewirkungen von Betriebsgründungen wird andererseits deutlich, daß die von Männern initiierten Betriebe eher zusätzliche Arbeitsplätze schaffen.

\section{Ergänzende Erfolgsindikatoren für Frauen- und Männerbetriebe}

Zur Abrundung des Bildes der Unterschiede der Entwicklung von Frauen- und Männerbetrieben im Zeitablauf soll abschließend noch ein Blick auf einige weitere (zum Teil auf subjektiven Einschätzungen beruhende) Erfolgsmaße der untersuchten Betriebsgründungen geworfen werden. Um dabei die im Aggregat geschlechtsspezifisch unterschiedlichen Bestandschancen der Betriebe zu kontrollieren, werden diese ergänzenden Erfolgsindikatoren getrennt für die Survivor-Betriebe einerseits und die abgemeldeten Betriebe andererseits betrachtet.

Die wesentlichen Ergebnisse dieses letzten Untersuchungsschrittes sind in Tabelle 6 zusammenge-

\footnotetext{
${ }^{6}$ Auf eine inhaltliche Kommentierung der Effekte der verschiedenen Kontrollvariablen (Humankapitalausstattung und Betriebsmerkmale) in den Modellen 2 von Tabelle 5 sei verzichtet, zumal die Gesamterklärungskraft der Modelle recht bescheiden ist.

${ }^{7}$ Da von gleichen Bestandschancen und ähnlichen Chancen einer Umsatzsteigerung nur unter der c. p.-Bedingung gesprochen werden kann und da wir. bei der Analyse des Überlebensprozesses und des Beschäftigten- bzw. Umsatzzuwachses unterschiedliche Gruppen betrachten (alle Betriebe versus Survivor-Einheiten), bleibt die angesprochene Argumentation zugegeben spekulativ.
}

faßt. Überblicken wir zunächst die Befunde für die Survivor-Einheiten in Tabelle 6, ergibt sich eine Bestätigung und zusätzliche Unterstützung der Ergebnisse des vorangehenden Abschnitts: Auch subjektiv deuten die Männer häufiger als die Frauen die Entwicklung ihres Betriebes retrospektiv im Sinne eines "Aufwärtstrends“. ${ }^{8}$ Weiterhin haben signifikant mehr Gründer als Gründerinnen innerhalb des Beobachtungszeitraums ihre Geschäftsräume erweitert und ihr Anfangs- bzw. Betriebskapital aufgestockt. Mit insgesamt $21 \%$ überraschend hoch ist der Anteil derer, die - obwohl ihr Betrieb ja inzwischen mehr als vier Jahre besteht angeben, daß sich der Betrieb bisher zu keinem Zeitpunkt rentiert hat bzw. Gewinne erbrachte. An dieser Stelle deutet sich eine beträchtliche Standfestigkeit der Gründer/innen an, die entweder die Substanz aufzehren oder durch Familie und/oder Partner unterstützt werden. Der Anteil der Frauen, die sich noch immer in der „Durststreckenphase“ ihres Betriebes befinden, ist dabei mit $30 \%$ deutlich höher als der Anteil bei den Männern mit $18 \%$. Der faktisch wohl in vielen Fällen nicht ganz gerechtfertigte Optimismus findet seinen Niederschlag auch darin, daß - von den Frauen etwas seltener als von den Männern - die Entscheidung der Betriebsgründung im nachhinein überwiegend nicht bereut wird und $\mathrm{da} \beta$ - von den Männern deutlich häufiger als von den Frauen für die Zukunft ein Wachstum bzw. eine Expansion des Betriebes erwartet wird.

Hinsichtlich der Geschlechtsdifferenzen deutlich andere Befunde erhalten wir für die abgemeldeten Betriebe. Nur bei einem der sieben Maße ergeben sich geschlechtsspezifische Unterschiede. Trotz Abmeldung sehen knapp 30\% der Gründerinnen und Gründer die Entwicklung ihres ehemaligen Betriebes als einen (vermutlich aber nicht ausreichenden) „Aufwärtstrend“. Bei der insgesamt wiederum erstaunlich hoch liegenden Bereitschaft zur nochmaligen Gründung zeigt sich für die Männer ein signifikant geringerer "Entmutigungseffekt", d. h. ihre Bereitschaft zur nochmaligen Gründung ist wesentlich höher. Sowohl etwa die Hälfte der Frauen als auch der Männer haben durch das

\footnotetext{
${ }^{8}$ Hierbei wurden die Befragten gebeten, aus einer Serie von Graphiken, die typische Verläufe von Betrieben im Zeitablauf darstellten, die für ihren Betrieb am ehesten zutreffende Graphik auszuwählen. Gegebenenfalls konnten die befragten Personen auf einem Beiblatt zum Interview auch eine eigene betriebliche „Entwicklungsgraphik" erstellen.
} 
Tabelle 6 Ergänzende Erfolgsindikatoren für Frauen- und Männerbetriebe.

\begin{tabular}{|c|c|c|c|c|c|c|}
\hline \multirow[b]{2}{*}{ Erfolgsindikatoren } & \multicolumn{3}{|c|}{ Survivor-Betriebe } & \multicolumn{3}{|c|}{ Abgemeldete Betriebe } \\
\hline & $\begin{array}{l}\text { Frauen } \\
(n=270)\end{array}$ & & $\begin{array}{l}\text { Männer } \\
(n=945)\end{array}$ & $\begin{array}{l}\text { Frauen } \\
(n=211)\end{array}$ & & $\begin{array}{l}\text { Männer } \\
(n=423)\end{array}$ \\
\hline $\begin{array}{l}\text { Subjektive Einschätzung der Betriebsentwicklung } \\
\text { als "Aułwärtstrend" }\end{array}$ & $64 \%$ & * & $75 \%$ & $29 \%$ & & $28 \%$ \\
\hline Geschäftsräume wurden erweitert & $21 \%$ & ** & $31 \%$ & $3 \%$ & & $5 \%$ \\
\hline Anlagen-/Betriebskapital wurde aufgestockt & $16 \%$ & * & $25 \%$ & $3 \%$ & & $4 \%$ \\
\hline $\begin{array}{l}\text { Betrieb hat sich bisher zu keinem Zeitpunkt rentiert } \\
\text { und Gewinne gebracht }\end{array}$ & $30 \%$ & ** & $18 \%$ & $59 \%$ & & $59 \%$ \\
\hline Person würde nochmals einen Betrieb gründen & $85 \%$ & * & $92 \%$ & $65 \%$ & ** & $79 \%$ \\
\hline $\begin{array}{l}\text { Betrieb wid in den nächsten Jahren wachsen } \\
\text { und expandieren }\end{array}$ & $45 \%$ & ** & $63 \%$ & & & \\
\hline $\begin{array}{l}\text { Betreiben der Firma war mit persönlichen, } \\
\text { finanziellen Verlusten verbunden }\end{array}$ & & & & $50 \%$ & & $54 \%$ \\
\hline $\begin{array}{l}\text { Betreiben der Firma brachte finanzielle Verluste } \\
\text { für andere (Geschäftspartner, Kreditgeber usw.) }\end{array}$ & & & & $14 \%$ & & $14 \%$ \\
\hline
\end{tabular}

\footnotetext{
** Unterschied zwischen den Subgruppen (Frauen-Männer) ist signifikant auf dem 1\%-Niveau; * signifikant auf dem 5\%-Niveau; aufgrund fehlender Werte bei den Aufgliederungsmerkmalen vermindern sich in den beiden Subgruppen zum Teil die Fallzahlen geringfügig.
}

Betreiben der Firma persönliche, finanzielle Verluste erlitten. Finanzielle Verluste für andere (Geschäftspartner, Kreditgeber usw.) gab es in $14 \%$ der Abmeldefälle. Das weitgehende Fehlen geschlechtsspezifischer Differenzen bei den abgemeldeten Betrieben kann dergestalt interpretiert werden, da $B$ in erster Linie betriebliche und ökonomische Faktoren, nicht jedoch die Zugehörigkeit zu einer Geschlechtsgruppe für die BetriebsschlieBung wichtig sind. Vermutlich befinden sich die Betriebe von Männern und Frauen in ähnlichen Zwangslagen, wenn die Entscheidung zu einer $\mathrm{Ab}$ meldung des Betriebes gefällt wird.

\section{Schlußbemerkungen}

Welches Fazit können wir nun mit Blick auf die beiden Ausgangsfragestellungen unserer Arbeit (,Selbständigkeit als erfolgversprechende Alternative für Frauen?“ und „Frauen als die schlechteren Unternehmer?") ziehen? Wie vielleicht von Anfang an nicht anders zu erwarten, läßt sich eine Antwort nicht ohne "Wenn und aber" geben. Sowohl aufgrund ihrer Humankapitalausstattung (allgemeines und spezifisches, unternehmerisches $\mathrm{Hu}$ mankapital) als auch aufgrund der Art der Betriebe, die sie gründen, treten Frauen mit schlechteren Ausgangsvoraussetzungen in die berufliche Selb- ständigkeit ein. Die Folge ist, daß die Bestandsund Überlebenschancen ihrer Betriebe im Aggregat ungünstiger sind als die der Männer. Würden jedoch die Frauen mit den gleichen Humankapitalressourcen wie die Männer den Weg in die Selbständigkeit beschreiten und/oder die gleiche Art von Betrieben gründen, gäbe es keine geschlechtsspezifisch unterschiedlichen Bestandschancen der Betriebe. Im Vergleich zu abhängigen Beschäftigungsverhältnissen, für die man in der Regel auch bei Kontrolle anderer, relevanter Faktoren einen eigenständigen Geschlechtseffekt findet, spricht dies zugunsten der Selbständigkeitsoption für Frauen. Frauen gründen ihre Betriebe vielfach von Anfang an mit bescheideneren Ambitionen und moderateren Ansprüchen als die Männer. Wie wir feststellen konnten, findet dies seine Fortsetzung in der Entwicklung der Betriebe im Zeitablauf. Die Entscheidung, zusätzliche Mitarbeiter/innen einzustellen (was häufig identisch ist mit dem Schritt von der Ein-Personen-Firma zum „SmallEmployer"), fällt Frauen offensichtlich schwerer als Männern. Interessant erscheint nicht zuletzt die Beobachtung, daB Frauen nach einer fehlgeschlagenen Betriebsgründung seltener als Männer zu einer nochmaligen Gründung bereit sind. Die häufigere Entmutigung und das geringere Aspirationsniveau von Frauenbetrieben sollten allerdings nicht im Sinne einer Geschlechterpsychologie in- 
terpretiert werden. Sie sind nicht mehr als eine Reaktion auf die Kontextbedingungen, die Frauen in der Phase der Gründung und in der Phase der Etablierung ihrer Betriebe vorfinden.

Wenngleich damit Frauen durch den Schritt in die berufliche Selbständigkeit insgesamt immerhin einige der Hürden auf dem allgemeinen Arbeitsmarkt umgehen können, verbleiben zahlreiche Probleme, die durchaus mit den Schwierigkeiten im Bereich der abhängigen Beschäftigung vergleichbar sind. Mit dem Schritt in die Selbständig. keit entfallen zwar der Arbeitgeber und der betriebliche Kontext, gleichzeitig jedoch kommen andere Akteure und Mechanismen ins Spiel, über die geschlechtsspezifische Rollenerwartungen wirksam werden (können). So z.B. berichten Gründerinnen überdurchschnittlich häufig von Schwierigkeiten bei der Beschaffung von Bankkrediten in der Anlaufphase ihrer Betriebe (dazu z. B. Bischoff 1985; Wloch/Ambos 1986). Im Fall der Einstellung männlicher, aber auch weiblicher Arbeitnehmer scheinen Frauen eher auf Führungsprobleme zu stoßen als "männliche Chefs“ (dazu z. B. Hennig/Jardim 1987; Assig et al. 1986). Wenn Gründerinnen hierauf dergestalt reagieren, daß sie von vorneherein keine Angestellten einplanen, schließen sie Expansionsmöglichkeiten weitgehend aus, und wenn sie nur Frauen einstellen, begrenzen sie ihr Arbeitskräftereservoir. Probleme ergeben sich auch im Bereich der Kunden- und Lieferantenbeziehungen (dazu z. B. Wloch/Ambos 1986). Abgesehen davon, daß die Gründerin gelegentlich mit der Sekretärin verwechselt wird bzw. für eine Angestellte gehalten wird, werden in diesen Beziehungen vielfach noch die klassischen Rollenvorstellungen und Stereotypen aktualisiert. Nicht zuletzt sind es zum Teil auch selbstauferlegte Beschränkungen, über die sich Frauen den Weg in die erfolgreiche Unternehmerrolle versperren. An dieser Stelle könnten die Frauen wohl am ehesten ansetzen, die berufliche Selbständigkeit - im Sinne einer individuellen Handlungsstrategie - noch stärker zu einer erfolgversprechenden Alternative zur abhängigen Beschäftigung bzw. zu anderen Lebens- und Arbeitsformen zu machen.

\section{Literatur}

Aldrich, J. H./Nelson, F. D., 1984: Linear Probability, Logit, and Probit Models. Newbury Park: Sage.

Ambos, I., 1989: Frauen als Unternehmerinnen und die Charakteristik ihrer Betriebe. Bielefeld: Kleine Verlag.

Assig, D., 1987: Mut gehört dazu. Informationen für Frauen, die beruflich selbständig sind oder werden wollen. Reinbek: Rowohlt.
Assig, D./Gather, C./Hübner, S., 1985: Voraussetzungen, Schwierigkeiten und Barrieren bei Existenzgründungen von Frauen. Berlin: Senator für Wirtschaft und Arbeit.

Assig, D./Gather, C./Hübner, S., 1986: Bruch mit der traditionellen Frauenrolle - Bericht über Existenzgründerinnen in Berlin. ifg - Institut für Frauenforschung, Heft 3: 34-45.

Autorinnengemeinschaft (Hg.), 1989: Arbeitsmarkt und Frauenerwerbsarbeit. SAMF-Arbeitspapier Nr. 198916. Paderborn.

Autorinnengemeinschaft (Hg.), 1990: Erklärungsansätze zur geschlechtsspezifischen Strukturierung des Arbeitsmarktes. SAMF-Arbeitspapier Nr. 1990-1. Paderborn.

Becker, G. S., 1975: Human Capital. 2d ed. Chicago: University of Chicago Press.

Beck-Gernsheim, E., 1976: Der geschlechtsspezifische Arbeitsmarkt. Frankfurt: Aspekte.

Bischoff, S., 1985: Kreditwürdigkeit - Ein Problem für Unternehmensgründerinnen? Das neue Erfolgs- und Karrierehandbuch für Selbständige und Unternehmer, Heft 10: 595-599.

Blau, F. D./Ferber, M. A., 1986: The Economics of Women, Men, and Work. New York: Prentice Hall.

Blossfeld, H.-P., 1985: Bildungsexpansion und Berufschancen. Frankfurt: Campus.

Blossfeld, H.-P., 1987: Labor Market Entry and the Sexual Segregation of Careers in the Federal Republic of Germany. American Journal of Sociology 93: 89-118.

Blossfeld, H.-P., 1989: Kohortendifferenzierung und Karriereprozeß. Frankfurt: Campus.

Blossfeld, H.-P./Hamerle, A./Mayer, K. U., 1986: Ereignisanalyse. Frankfurt: Campus.

Bögenhold, D., 1987: Der Gründerboom. Frankfurt: Campus.

Boswell, J, 1972: The Rise and Decline of Small Firms. London: Allen and Unwin.

Bock-Rosenthal, E./Haase, C./Streeck, S., 1978: Wenn Frauen Karriere machen. Frankfurt: Campus.

Brüderl, J./Preisendörfer, P./Baumann, A., 1991: Determinanten der Überlebenschancen neugegründeter Kleinbetriebe. Mitteilungen aus der Arbeitsmarktund Berufsforschung 24: 91-100.

Bühler, C., 1991: Empirische Befunde zum Sozialprofil von Unternehmensgründern. Diplomarbeit am Münchner Institut für Soziologie. München.

Dahlke, G., 1986: Frauenerwerbstätigkeit im Spannungsfeld von Gleichberechtigung und Gleichstellung. Düsseldorf: Verlagsanstalt Handwerk.

Diekmann, A., 1985: Einkommensunterschiede zwischen Frauen und Männern. Forschungsberichte Nr. 212 und Nr. 214 des Wiener Instituts für Höhere Studien. Wien: Fachverlag für Wirtschaft und Technik.

Diekmann, A./Mitter, P., 1984: Methoden zur Analyse von Zeitverläufen. Stuttgart: Teubner.

Dittrich, H., 1985: Wege und Tips zur Existenzgründung. München: Humboldt. 
Engelbrech, G., 1989: Erfahrungen von Frauen an der „dritten Schwelle“. Schwierigkeiten bei der Wiedereingliederung aus der Sicht der Frauen. Mitteilungen aus der Arbeitsmarkt- und Berufsforschung 22: $100-113$.

Engelbrech, G., 1991: Frauenspezifische Restriktionen des Arbeitsmarkts - Situationsbericht und Erklärungsansätze zu Phasen des Berufsverlaufs anhand von IAB. Ergebnissen. S. 91-118 in: K. U. Mayer/J. Allmendinger/J. Huinink (Hg.), Vom Regen in die Traufe: Frauen zwischen Beruf und Familie. Frankfurt: Campus.

Gärtner, H. J./Krebsbach-Gnath, C., 1987: Berufliche Qualifizierung von Frauen zur Verbesserung ihrer Berufschancen bei der Einführung neuer Technologien. Stuttgart: Kohlhammer.

Handl, J., 1986: Führt die Angleichung der Bildungschancen zum Abbau geschlechtsspezifischer beruflicher Segregation? Eine einfache Frage, aber kontroverse Antworten. Zeitschrift für Soziologie 15: 125-132.

Hellmich, A., 1986: Frauen zwischen Beruf und Familie. Eine Untersuchung über Voraussetzungen und Nutzen einer Berufskontaktpflege von Frauen in der Familienphase. Stuttgart: Kohlhammer.

Hennig, M./Jardim, A., 1987: Frau und Karriere. Erwartungen, Vorstellungen, Verhaltensweisen. Reinbek: Rowohlt.

Hofmann, M., 1986: Existenzgründung: Ein praktischer Ratgeber für alle, die sich selbständig machen wollen. Heidelberg: Decker und Müller.

Kirsch, C./Lühder, K., 1991: Existenzgründungen durch Frauen, Aus Politik und Zeitgeschichte (Beilage zur Wochenzeitschrift Das Parlament), B14-15/91, 29. 3. 1991: 25-33.

Klandt, H., 1984: Aktivität und Erfolg des Unternehmensgründers. Bergisch-Gladbach: Josef Eul.

Krüger, H., 1989: Geschlecht als Strukturkategorie im Bildungssystem: Alte und neue Konturen geschlechtsspezifischer Diskriminierung. S. 63-101 in: Autorinnengemeinschaft ( $\mathrm{Hg}$.), Arbeitsmarkt und Frauenerwerbsarbeit. SAMF-Arbeitspapier Nr. 1989-16. Paderborn.

Laband, D. N./Lentz, B. F., 1985: The Roots of Success. Why Children Follow in Their Parents' Footsteps. New York: Praeger.

Lappe, L., 1981: Die Arbeitssituation erwerbstätiger Frauen. Geschlechtsspezifische Arbeitsmarktsegmentation und ihre Folgen. Frankfurt: Campus.

Marini, M. M., 1989: Sex Differences in Earnings in the United States. Annual Review of Sociology 15: $343-380$.
Markmann, E., 1988: Frauen machen sich selbständig. München: „Brigitte“-Buch.

Mayer, K. U., 1991: Berufliche Mobilität von Frauen in der Bundesrepublik Deutschland. S. 57-90 in: K. U. Mayer/J. Allmendinger/J. Huinink (Hg.), Vom Regen in die Traufe: Frauen zwischen Beruf und Familie. Frankfurt: Campus.

Mayer, K. U./Allmendinger, J./Huinink, J. (Hg.), 1991: Vom Regen in die Traufe: Frauen zwischen Beruf und Familie. Frankfurt: Campus.

Metzler, G., 1986: Frauen, die es geschafft haben. Portraits erfolgreicher Karrieren. 2. Aufl. Düsseldorf: Econ.

Mincer, J., 1974: Schooling, Experience and Earnings. New York: Columbia University Press.

Ostner, I., 1990: Das Konzept des weiblichen Arbeitsvermögens. S. 22-39 in: Autorinnengemeinschaft (Hg.), Erklärungsansätze zur geschlechtsspezifischen Strukturierung des Arbeitsmarktes. SAMF-Arbeitspapier Nr. 1990-1. Paderborn.

Picot, A./Laub, U.-D./Schneider, D., 1989: Innovative Unternehmensgründungen. Berlin: Springer.

Preisendörfer, P.Noss, T., 1990: Organizational Mortality of Small Firms: The Effects of Entrepreneurial Age and Human Capital. Organization Studies 11: 107-129.

Preisendörfer, P./Ziegler, R., 1990: Adressenaktualisierung zum Feldverlauf einer Studie über Gründung und Erfolg von Kleinbetrieben. ZUMA-Nachrichten, Nr. 27: 93-108.

Rabe-Kleberg, U., 1987: Frauenberufe - Zur Segmentierung der Berufswelt. Bielefeld: Kleine Verlag.

Schlemper-Kubista, A./Wollrab, H., 1980: Die wirtschafts- und gesellschaftspolitische Bedeutung der selbständigen Unternehmerinnen und mitarbeitenden Unternehmerfrauen. Göttingen: Schwartz.

Tölke, A., 1989: Lebensverläufe von Frauen. Familiäre Ereignisse, Ausbildung, Erwerbsverhalten. München.

Willms-Herget, A., 1985: Frauenarbeit. Zur Integration der Frauen in den Arbeitsmarkt. Frankfurt: Campus.

Wloch, E./Ambos, I., 1986: Erschließung neuer beruflicher Arbeitsfelder und Tätigkeiten für Frauen - Frauen als Selbständige. ifg - Institut für Frauenforschung, Heft 3: 1-33.

Wloch, E./Siegel, 1., 1988: ErschlieBung neuer beruflicher Arbeitsfelder für Frauen - Frauen als Selbständige. Teil II: Frauen in Freien Berufen. ifg - Institut für Frauenforschung, Heft 1/2: 131-149. 